\title{
LA LECTURA DE LOS CLÁSICOS DE LA FILOSOFÍA EN LA UNIVERSIDAD ${ }^{1}$ The Reading of the Classics of Philosophy In the University
}

\author{
Alfonso de La Cruz Martínez $^{2}$
}

Fecha de recepción: 18 de noviembre de 2015

Fecha de aceptación: 2 de diciembre de 2015

SUMARIO: 1. Introducción; 2. ¿A qué llamamos clásicos de la filosofía?; 3. La filosofía como orientadora del saber; 4. La filosofía como necesidad académica y social; 5. La filosofía en el campo de la academia; 6. Retos del abordaje de los clásicos de la filosofía;

7. Conclusión; 8. Referencias bibliográficas.

\footnotetext{
${ }^{1}$ El presente artículo es derivado de la investigación titulada La lectura de los clásicos de la filosofía en la universidad. Esta investigación fue financiada con recursos propios, y en su desarrollo el autor de este artículo actuó como investigador principal.

${ }^{2}$ Abogado, Magister en Derecho Público, Doctorando en Ciencias Jurídicas por la Universidad de Granada, con estudios en Gobernanza, Filosofía y Humanidades. Facilitador del programa Eficacia Comunicativa ECO en la Universidad del Norte; Profesor Catedrático Universidad del Norte. Correo: alfonsodelacruz@uninorte.edu.co.
} 


\section{COMO SE CITA ESTE ARTÍCULO (APA 6)}

De La Cruz Martínez, Alfonso (2016). La lectura de los clásicos de la filosofía en la universidad. Revista Jurídica Mario Alario D’Filippo, VIII (15), pág 84-97.

\section{RESUMEN}

El presente artículo pretende sustentar la importancia de la lectura de los clásicos de la filosofía en la formación universitaria. El autor sostiene que es preciso brindarles a los estudiantes universitarios las herramientas de análisis pertinentes, tanto desde el ámbito profesional como personal, para afrontar los complejos problemas que surgen en los distintos contextos sociales, los cuales exigen necesariamente una reflexión filosófica acorde con sus particularidades. Esta sustentación se realiza desde una metodología analítica, la cual incluye un estudio de la importancia de la filosofía, mostrar su relevancia en los ámbitos académico y social, y por ultimo entender la significación del estudio de los textos clásicos de la filosofía en el saber universitario.

\section{PALABRAS CLAVES}

Filosofía, pensamiento, universidad, perfil profesional.

\section{ABSTRACT}

This article aims to support the importance of reading the classics of philosophy in university education. The author argues that it is necessary to provide university students with the relevant analytical tools, both from a professional and personal level, to face the complex problems that arise in the different social contexts, which necessarily require a philosophical reflection according to their particularities. This support is carried out from an analytical methodology, which includes a study of the importance of philosophy, show its relevance in the academic and social spheres, and lastly to understand the significance of the study of classical texts of philosophy in university knowledge.

\section{KEYWORDS}

Philosophy, thought, university, professional profile. 
Lo que más merece pensarse en nuestro tiempo problemático es el hecho de que no pensamos.

(Heidegger, 2005)

\section{INTRODUCCIÓN}

La lectura de los textos clásicos de la filosofía debe convertirse en parte fundamental de la formación de los estudiantes universitarios, no solamente de aquellos que se inscriben en los saberes de las ciencias sociales, sino para todos aquellos que pretendan adquirir una estructura de pensamiento sólida; pero más allá, para quienes consideren necesaria una formación integral como ciudadanos dotados de un sentido profundamente humanista con qué afrontar los problemas de la postmodernidad.

Por tanto, el cultivo del pensamiento filosófico debe significar para todo estudiante un modelo de forma de pensamiento formal y material, centrado en las grandes preocupaciones del hombre, y direccionado por aquellos referentes históricos que, más allá del tiempo, siguen sonando en nuestros días como verdaderos clásicos del pensamiento universal.

Pero, paradójicamente, en los actuales sistemas y programas de educación superior existe un temor latente e infundado de los estudiantes de leer y de los profesores de poner a leer a los clásicos de filosofía. La filosofía es vista a la distancia por parte del estudiante; a sus ojos, pareciese ser inalcanzable. Por otra parte, los profesores recelosos de una posible complejidad de los textos filosóficos se abstienen de incluirlos en sus programas académicos. La filosofía de esta manera se ha marginalizado del ámbito académico.

Se debe comenzar a repensar esta concepción de la filosofía y retomarla al interior de los programas académicos, especialmente en centros de avanzada como la universidad. Debemos preguntarnos sobre qué tipo de ciudadanos están tratando de producir nuestros establecimientos superiores y en qué medida lo están logrando (Nussbaum, 2005). Los problemas del mundo moderno hoy pareciesen versar justamente sobre el olvido de las cuestiones fundamentales que plantea la filosofía: la ética frente a la corrupción; la relación consigo mismo, con los demás y con el mundo, siempre en escenarios llenos de tensiones políticas y ambientales; el papel mismo del hombre en la historia y su legado individual en la sociedad en que vive; y claro, toda una serie de cuestiones académicofilosóficas que terminan por aunar valor a la formación profesional ya consistente de la lectura en filosofía.

\section{2. ¿A QUÉ LLAMAMOS CLÁSICOS DE LA FILOSOFÍA?}

Partamos del hecho de que en el quehacer filosófico rara vez hay un consenso total; de hecho, ni siquiera lo hay acerca de la definición de filosofía. Algunos consideran que es 
una disciplina; otros, que es un conjunto de reflexiones sin una unión sistemática; unos la consideran como un acumulado de formas de pensar; otros, como una manera de teorizar, entre otras varias posturas.

Con la pregunta "¿Qué es lo que se tiene que estudiar al estudiar filosofía?" sucede más o menos lo mismo. Algunos sugieren que se deben estudiar las ideas con independencia del contexto, otros arguyen que sin el contexto histórico la comprensión de una tesis filosófica es imposible, y unos terceros sostienen que el contexto es importante, pero prescindible en ocasiones. $Y$ no es de sorprender que, si se pregunta a distintos filósofos por las características que debería tener un texto para ser filosófico, las respuestas variarían.

No obstante, para el fin de este artículo bastará con una noción preliminar de lo que estamos hablando. Basta pues, entender a la filosofía como un conjunto de problemas y los intentos de reflexión y solución que giran en torno a estos. Intuitivamente, la filosofía abarca preguntas acerca de la ética, la metafísica, la epistemología, la lógica y otros variados temas. Se tiene de esta manera un indicio de las características de los clásicos, pero estos parece que integran todos estos problemas en una unidad a preguntas diversas. Es el tipo de respuestas que se busca. Los clásicos de la filosofía no indagan por descripciones ni por contestaciones superficiales. Los clásicos de la filosofía, pregúntese por lo que se pregunte, buscan los fundamentos de ello, y de ahí su carácter imperecedero en la historia.

Sin embargo, y muy a pesar de que exista un acercamiento al ámbito de la filosofía y a las preguntas que esta genera, se necesita ir más allá del estudio de su historia o de sus autores; salir de la apariencia que nos simula como estudiosos insaciables de la ciencia y la filosofía. De la mano de los clásicos se requiere aprender a pensar por nosotros mismos (Heidegger, 2005). Este es el verdadero llamado de la filosofía.

Por ello, para contestar a estas preguntas los filósofos se forman teorías: generan conceptos y relaciones entre esos conceptos, para dar cuenta de aquello que quieren explicar. Por ejemplo, la teoría de las ideas de Platón busca explicar lo que es el bien, cómo es posible el conocimiento, el mundo físico, la sociedad, etc. Y para explicar estas cosas, Platón utiliza conceptos como "ideas", "copias", "formas", etc. Unas nociones, pues, explican el problema que se intenta tratar.

En este aspecto, la filosofía es similar a la ciencia: dada una pregunta, se generan teorías para contestarlas. Por ejemplo, la teoría de la evolución surgió para explicar los rasgos de los seres humanos y contestar la pregunta sobre las formas de vida que se observan. La teoría del big-bang contesta la pregunta sobre el origen del universo. De hecho, lo que actualmente conocemos como ciencia era, en algún punto, filosofía. 
Ejemplo notable de lo anterior es el hecho de que el trabajo de Newton se consideraba "filosofía natural". Otro ejemplo, es que la forma primitiva de la psicología era en realidad parte de la filosofía. Muchas de las preguntas que se contestan actualmente en ciencia, son preguntas que la filosofía se había formulado; el origen del cosmos es uno de esos asuntos. Así pues, ciencia y filosofía tienen al menos un propósito común: explicarnos el mundo.

Partiendo del propósito común que establecemos entre la filosofía y la ciencia, se podría decir que en la era de la hiperespecialización, se encuentran más respuestas en la ciencia que en la filosofía, que pareciera se dedica a cuestionarnos y no a respondernos, $y$, sin embargo, quizá el hombre hasta ahora, desde siglos, ha actuado ya demasiado y pensado demasiado poco (Heidegger, 2005). Es decir, podemos dejar de lado el estudio de los clásicos de la filosofía porque las ingenierías pueden responden a las preguntas, pero sobre todo a las necesidades de nuestros días que la filosofía no puede, y nunca podrá. Dicho de otro modo ¿para qué filosofía dentro del estudio científico?

\section{LA FILOSOFÍA COMO ORIENTADORA DEL SABER}

Los clásicos tienen un interés universal no sólo en entender el mundo, sino también en cambiarlo. Su conocimiento esencial apenas descrito, trasciende tiempo y situaciones. La filosofía política o moral, por ejemplo, busca mejorar las condiciones de vida de las personas. La democracia en la filosofía de los griegos y latinos, o el comunismo en la filosofía de Karl Marx, son ejemplos de esta búsqueda por el bien de las personas.

La ciencia no puede reemplazar a la filosofía, mientras que tanto la filosofía como la ciencia se preocupan por "¿qué son las cosas?", sólo la filosofía habla de "cómo deberían ser las cosas". La ciencia sabe qué se necesita para poder construir una bomba nuclear, pero no puede decir si debería usar o no, ese espacio es para la filosofía. Y este espacio es de vital importancia para los seres humanos, pues el espacio ético permea todas nuestras actividades. Los médicos frecuentemente se ven ante preguntas que sus estudios en medicina no pueden ayudarles a solventar, al igual que los abogados y cualquier persona que se vea en necesidad de decidir. En estos asuntos tan importantes y comunes, es la filosofía, no la ciencia, la que puede ayudar; lo que revela el carácter orientador de la filosofía. En este sentido (Narvaja, Stefano, \& Pereira, 2004):

(...) Desde este enfoque, se hace hincapié en las representaciones (o sea, en las ideas, en el conjunto de creencias y valores) sobre el mundo que poseen los grupos sociales, ya que se considera que éstas son orientadoras del tipo de prácticas que encaran los grupos, y del modo en que deben llevarlas a cabo. Así, en cuanto a la lectura, se ha señalado que las representaciones acerca de lo que es leer difieren en los distintos grupos sociales, y en las distintas esferas de la praxis social, e inciden en el modo en que los sujetos encaran sus lecturas, en la finalidad que les otorgan, en los objetos que seleccionan para ser leídos, e incluso, en las operaciones cognitivas que despliegan para hacerlo, entre otros. 
Desde esta perspectiva, presente en los estudios realizados por la historia social de la lectura, esta es concebida como una praxis social, determinada histórica y culturalmente. (Pág. 9).

Otro motivo por el cual la ciencia no puede reemplazar a la filosofía es este: la filosofía estudia temas que la ciencia simplemente no puede contestar. "¿Qué es el arte?", "¿en qué consiste el significado?”, “¿a qué acción social deberíamos darle prioridad?”, “¿Qué es la conciencia?", “¿Qué es la realidad?” son ejemplos de aquellas preguntas que no se pueden, por principio, contestar en un laboratorio. $Y$ son, además, preguntas que reconocemos importantes al tratar de aspectos que nos incumben como personas. Así pues, la facultad de filosofía no se puede reemplazar simplemente con una de ingenierías. Se revela entonces otro carácter de la filosofía: estudiar filosofía es cultivar un sentido de humanidad.

Es cierto que la ciencia ha logrado dar con el genoma humano: con aquella estructura química que nos diferencia de otros organismos. También es cierto que ha mejorado los aspectos del cuerpo humano, pues, por ejemplo, se ha extendido el tiempo de vida de los seres humanos gracias a los avances de la medicina. Estas mejoras son innegables. No obstante, la ciencia es inerte en los asuntos del deber. No hay gen de lo correcto, no hay magnitud física de ello. Los avances de la ciencia sirven tanto para mantener la vida (medicinas) como para exterminarla (armas). La ingeniería no distingue entre hacer un puente o un campo de concentración. Por eso, la filosofía es la que puede tratar mejor que cualquier otra disciplina la pregunta de hacía donde debe ir la sociedad.

En ese sentido, la ciencia nos da el vehículo y el sistema de navegación: funcionales y eficientes. Pero la filosofía, es la que determina lo que se ha de buscar y los motivos para hacerlo, es la que nos traza el destino. La época moderna se ha enfocado en optimizar el consumo de combustible, mejorar la aerodinámica, y hacer más preciso al sistema de geolocalización; pero aún no se ha preguntado a dónde vamos. Hace falta, pues, filosofía. No es que se necesite a la una y se pueda prescindir de la otra: se necesitan a ambas dentro de la sociedad ${ }^{3}$.

Un tercer punto de vista punto de vista para negarle importancia a la filosofía es el que afirma que, dada a su antigüedad, no nos sirve para tratar los problemas del presente. Este argumento es simplemente falso: la época en que surgió no define el valor de una teoría. Tomemos como ejemplo el teorema de Pitágoras. Los conceptos que explican la relación entre los lados de un triángulo rectángulo surgieron hace más de dos milenios, y no por ello se han vuelto inútiles.

Un cuarto argumento para decir que la filosofía es inútil, es el que sostiene que la filosofía es un conjunto de teorías que han resultado equivocadas en ocasiones. A este

${ }^{3}$ Acerca de la relación entre filosofía y sociedad, véase las secciones 3 , y 4 de este artículo. 
razonamiento se le objeta que el hecho de que una teoría haya resultado falsa en algunas de sus predicciones, no implica que la teoría es inútil.

Por ejemplo, si usáramos la física newtoniana para ajustar la hora en los satélites, los relojes de estos aparatos estarían seriamente desajustados, y, por ende, nuestros sistemas de navegación serían un caos. Es decir, la teoría está equivocada en algunas de sus predicciones. La teoría que permite ajustar correctamente el tiempo en los satélites es la teoría de la relatividad. No obstante, no por estos errores la física de newton se ha tornado inútil: sirve para explicar lo que sucede a nuestra escala. Es tan útil, que la teoría que usamos en nuestras actividades cotidianas es la de Newton, no la de Einstein.

Así entonces, se ha mostrado la relación entre la ciencia y la filosofía, mostrando que la primera no puede reemplazar a la segunda. La principal conclusión de esta sección es que no hay razones para creer que la filosofía es inútil. Esto implica, que no hay razones para creer que no se necesita estudiar filosofía en las universidades.

\section{LA FILOSOFÍA COMO NECESIDAD ACADÉMICA Y SOCIAL}

Por lo que vimos en la sección anterior, podemos decir que hay al menos dos razones por las que la filosofía es útil: porque permite entender la realidad y porque permite, en ocasiones, moldearla. Estas razones se aplican también a la pregunta de por qué leer a los clásicos. En esta sección, daremos razones adicionales para estudiar filosofía y por tanto, razones de por qué estudiar a los clásicos.

¿De qué me va a servir leer a Platón en mi vida cotidiana? Directamente, es poco probable que sirva de algo. Pero indirectamente, las ventajas son innegables. Analicemos la siguiente analogía. Al año, alrededor del mundo se gastan millones de dólares en gimnasios. ¿Qué son estos sitios? Son sitios a los que pagas para poder esforzarte levantando y bajando pesos, corriendo sin llegar a ningún lado y realizando otras actividades que no se completan. ¿Sirve de algo pedalear sin moverse? Directamente, no. Es un gasto de energía innecesario. Indirectamente, los beneficios son innegables, se mejora el sistema cardiovascular, se baja de peso, se optimiza el metabolismo, se exige a los músculos, etc.

Análogamente, leer a los clásicos es una actividad que probablemente no ayude a alcanzar un objeto en concreto. Lo que sí hará con seguridad, será ejercitar el cerebro del lector. El ejercicio de buscar los razonamientos, las definiciones, las refutaciones, las relaciones entre ideas y todo lo demás que ofrece un clásico, genera una mente crítica y comprensiva. El lector se perfecciona a sí mismo mediante la actividad.

Se puede objetar diciendo que se puede leer cualquier cosa y que el beneficio sería el mismo. Es decir, no es necesario que sean textos filosóficos. La analogía del gimnasio también sirve para contestar a esta objeción. Levantar un lapicero no es lo mismo que 
levantar una mancuerna. Para que sea un ejercicio efectivo, hace falta que exija algún grado de esfuerzo, característica por los que los textos de filosofía son especialmente famosos.

En este sentido no se puede caer en el error de concebir la lectura, y en ese mismo modo, la escritura como meras habilidades cognitivas, desvinculada de cualquier lazo social, afectivo o personal. La lectura en este sentido errado estaría reducida a una especie de habilidad estática, acotada, delimitada: Sería una experiencia que es igual para todos, y que debe ser aprendida por una sola vez, de aplicación automática descontextualizada (Cassany \& Morales, 2009).

Una de las ventajas de realizar el ejercicio filosófico, es que se aprende poco a poco a razonar correctamente. Pues, como bien es sabido, la lógica es una de las ramas y herramientas de la filosofía. Razonar correctamente, es la base para argumentar correctamente. Por lo cual, si alguien en algún momento desea sostener que la filosofía es inútil, le será de utilidad leer a los clásicos para que su argumento sea tan sólido como sea posible.

El ejercicio filosófico, también trae como resultado el mejoramiento de la capacidad crítica. Esto quiere decir que se es capaz de evaluar de forma más precisa las cosas que se pretenden pasar por verdades. Y esto se logra buscando los fundamentos de lo que se afirma y la relación con hechos ya verificados. Aunque la capacidad crítica es útil en muy diversos ámbitos, es de especial utilidad en el ámbito político.

Un ejemplo en que brilló por su ausencia, fue en el plebiscito que se realizó en Colombia el 2 de octubre del 2016. El plebiscito pretendía permitir que las personas manifestaran su aprobación o desaprobación del acuerdo firmado entre el gobierno colombiano y las Fuerzas Armadas Revolucionarias de Colombia. El problema consistió en que la oposición al gobierno se encargó de realizar falsas afirmaciones acerca de las consecuencias de aceptar el acuerdo. Esto llevó a que se rechazara dicha propuesta. Ahora bien, la capacidad de analizar lo que otros dicen y ver si se fundan en verdades, es en sí un ejercicio crítico, y pudo haber evitado el engaño en el que cayeron millones de colombianos. Dicho de otro modo: de haberse estudiado más filosofía, los resultados del plebiscito pudieron haberse evitado.

A propósito de estos escenarios en contexto, que requieren de verdaderos lectores de realidades políticas y sociales, la teoría lingüística

(...) toma como unidad de análisis el texto en relación solidaria de mutua determinación con el contexto social en el que se produce. Define el texto como unidad real a través de la cual se negocia significado (...) y concibe el lenguaje como un sistema semiótico en relación de realización con otro más abstracto: el contexto (Moyano, 2007). 
Un corolario a esa capacidad crítica que se adquiere gracias a la filosofía, es la capacidad de pensar nuevas posibilidades. Pues, aunque ciencia y filosofía son muy cercanas, hay una gran diferencia entre ellas y es que la filosofía se toma en serio lo que podría haber sido, y lo que podría ser. Una persona que se ha entrenado en filosofía no solo entiende el momento histórico en el que vive, sino que, además, entiende que su momento podría ser distinto, que podría ser cambiado. Así pues, el entrenamiento en filosofía puede traer consigo una consciencia de la necesidad de acción y de cambio.

Estas ventajas que trae el estudio de la filosofía, conllevan a su vez a la formación de ciudadanos. Es decir, tener capacidad crítica y la capacidad de razonar apropiadamente son condiciones para ser un miembro ejemplar de la sociedad. No obstante, estas no son las únicas habilidades necesarias. También hace falta la capacidad propositiva y empatía. En los siguientes párrafos se argumentará a favor de que estas son habilidades propias de un ciudadano y que, además, se pueden obtener gracias a la filosofía.

Es necesario tener en cuenta que, aún en el ámbito académico, y por ello, en plena relación con la formación ciudadana, la presencia de la filosofía se articula lo más a menudo con las tradiciones culturales en las cuales se inserta. Limitar la presencia de la filosofía solo a materias designadas como "filosofía" es una trampa que hay que evitar en lo cultural. Muy a menudo, se imparten enseñanzas de teoría política, de religión, de éticas profesionales, así como de psicología social o de historia de las ideas que corresponden, plenamente, a conceptos o categorías filosóficas (UNESCO, 2011). Esto es de vital importancia en el momento de dilucidar que incluso la filosofía, tal vez en parte como la ciencia, también puede proponer soluciones a problemas sociales dados.

La capacidad de proponer es, dicho de modo simple, la capacidad de hallar posibles soluciones para un problema dado. Así, no solo es necesario saber criticar las posturas que puedan sostener ciertos actores políticos, sino que también es de vital importancia ser capaces de sugerir una opción mejor a la disponible (sobre un ejemplo de esto, se puede ver la sección 4 de este artículo). Para la democracia es necesaria la postulación de posturas.

La filosofía ayuda a adquirir esta capacidad, pues crear nueva filosofía es justamente eso: generar nuevas posturas para alimentar el debate. Estas posturas se construyen utilizando, claro está, las reglas del razonamiento correcto. Y leer filosofía, es también proponer algo: es proponer una interpretación (la sección 5 desarrolla un poco más el asunto de la lectura en filosofía). Por ende, el estudio de la filosofía puede ayudar con el desarrollo de esta habilidad necesaria para el ciudadano.

La habilidad de sentir empatía consiste en la capacidad de reconocerse a uno mismo en los demás, en tomar problemas e intereses ajenos como si fueran propios. Esta empatía va más allá de una identificación con los que son de nuestro propio grupo, es una empatía 
que incluye a los que no son "nosotros". Esto es de vital importancia en una democracia pues hay asuntos que no nos afectan directamente, pero que es nuestro deber hacer algo al respecto. Por ejemplo, la muerte de niños en condiciones de extrema pobreza. No nos afecta directamente, pues no somos esos niños y probablemente ni siquiera los conozcamos; pero nos compete tomar una posición porque tenemos la capacidad de ejercer un cambio.

¿Y cómo puede la filosofía ayudar a generar empatía? La filosofía busca verdades y deberes universales, que se apliquen con independencia de situaciones contingentes. Es decir, la filosofía tiene pretensiones de hablar acerca de TODOS y no sólo de algunos. En ese sentido, pensar filosóficamente puede ayudar con el desarrollo de la empatía.

Así pues, la filosofía nos ayuda a desarrollar al menos cuatro habilidades que son necesarias para la formación del ciudadano4: la capacidad de crítica, de razonamiento adecuado, de proposición y de empatía. Y, por ende, al ayudar a formar a los ciudadanos, es innegable la importancia que tiene la filosofía para la sociedad del presente (Nussbaum, 2005).

Hay otro gran grupo de razones por las cuales el estudio de los clásicos es importante: puesto que los clásicos moldearon a Occidente, entenderlos a ellos es entender entonces a parte de Occidente. Ejemplo de esta influencia es que los trabajos de Aristóteles por muchos siglos fueron el canon en los estudios. La física aristotélica fue reemplazada por la newtoniana, lo cual quiere decir que por más de un milenio Aristóteles fue utilizado para explicar el mundo.

Otro de los clásicos de la filosofía (y con clásicos hacemos referencia a los que son de lectura obligada, y no sólo a los antiguos) es Adam Smith, uno de los padres del capitalismo. Sin duda alguna, el capitalismo ha forjado muchos de los aspectos que tiene occidente. Así pues, entender al capitalismo puede dar cuenta de mucho de lo que pasa en el mundo; y para una mejor comprensión de este sistema económico conviene ir a sus bases: un filósofo.

Por otro lado, el comunismo también ha moldeado gran parte del globo. Entenderlo, pues, será entender gran parte de los movimientos revolucionarios y de las economías de países como Cuba y algunos de Asia. Y puesto que las bases del comunismo como se entiende intuitivamente se hallan en la obra del filósofo Karl Marx, se sigue que estudiar filosofía servirá para entender no solo a Occidente, sino también a lo que se le opone.

\section{LA FILOSOFÍA EN EL CAMPO DE LA ACADEMIA}

Se parte de la premisa de que la naturaleza de lo que debe ser aprendido (leer y escribir textos específicos de cada asignatura en el marco de las prácticas académicas disciplinares) exige un abordaje dentro del contexto propio de cada materia en la universidad (Carlino,

\footnotetext{
${ }^{4}$ Para una lectura distinta de estas habilidades, veasé (Nussbaum, 2005).
} 
2005). La filosofía impregna esta premisa y la llena de sentido no sólo académico sino también humanista. La labor universitaria tiene que ir más allá.

Sobre el aspecto político en Latinoamérica y la filosofía, se podría añadir una observación. Una de las ventajas del estudio de la filosofía, es la generación de nueva filosofía. Es decir, el estudio del pensamiento de otros estimula el pensamiento propio. Y esto hace falta, mucha falta, en el contexto latinoamericano. Pues, como se ha señalado en muchas instancias, la política y la economía que se incorpora en nuestros países son copias de las que se instauran en otros. Comunismo ni capitalismo ni las otras opciones parecen ajustarse a las condiciones de nuestros países, ¿por qué, entonces, no generar sistemas para nosotros mismos? El diseño de un nuevo sistema, es tarea de la filosofía pues sólo ella puede tratar acerca de cómo deberían ser las cosas. Y para propiciar un ambiente académico capaz de esto, es necesario impulsar el trabajo filosófico.

Ahora bien, cabe hacer explícito el hecho de que los beneficios expuestos no recaen únicamente sobre los estudiantes de filosofía. Y es, por tanto, importante notar que la filosofía es útil para todos los ciudadanos pues a todos nos conviene tener capacidad crítica y de razonar apropiadamente. Sabemos que nuestros campus están formando ciudadanos, y eso significa que debemos preguntarnos cómo debe ser un buen ciudadano de hoy y que debe saber. Y esta pregunta es claramente filosófica.

En este sentido,

(...) cobra especial importancia comprender la naturaleza de la interacción en el aula. No sólo en cuanto a los contenidos y las formas lingüísticas de los discursos que circulan en ella sino también los patrones de interacción y su relación con los procesos de aprendizaje (Barletta, 2015).

Dicho de otro modo: la filosofía nos debe decir qué tipo de personas es el que busca producir una universidad.

La pregunta por el tipo de personas que las universidades pretenden producir y la pregunta por si lo están logrando, son asuntos filosóficos con fuertes implicaciones socioculturales. Y es evidente que el tipo de ciudadanos que se debe intentar generar en las academias depende del tiempo en que se vive. Se sabe que estamos en una época de creciente diversidad cultural e internacionalización. Por tanto, la misión de la academia será en parte preparar ciudadanos aptos por un ambiente, entre otras cosas, diverso.

El nuevo énfasis en la Universidad de "diversidad" en los currículos de las escuelas superiores y universidades es, sobre todo, un modo de hacerse cargo de los nuevos requisitos de la condición de ciudadano de los deberes, derechos y privilegios que le son propios; un intento de producir adultos que pueden funcionar como ciudadanos no sólo de algunas regiones o grupos locales, sino también, y más importante, como ciudadanos de un mundo complejo e interconectado. 


\section{RETOS DEL ABORDAJE DE LOS CLÁSICOS DE LA FILOSOFÍA}

Pese a que a se ha argumentado por la importancia y necesidad vital de la filosofía no solamente en el ámbito académico, sino también con influencia en la vida en sociedad, existe una renuencia, o una marginalización de la misma. Hay un miedo de leer por los estudiantes, y hay un miedo de los profesores de poner a leer directamente a los clásicos de la filosofía. Esto se debe a que, como menciono anteriormente, la lectura de los textos filosóficos exige esfuerzo por parte del que se acerque a ellos. Este esfuerzo es el responsable de que la lectura de los clásicos, mejore a quién lo haga. No obstante, ¿en qué consiste propiamente este esfuerzo? ¿En qué consiste leer filosofía?.

Leer filosofía no es recibir, consumir, o adquirir la información que brinda el texto ${ }^{5}$. Leer es trabajar. Esto quiere decir que la lectura en filosofía implica la construcción de una interpretación por parte del lector. Esto último debido a que los códigos de un texto (sus palabras y frases) pueden cobrar significaciones diversas, y el trabajo interpretativo es justamente buscar las interpretaciones adecuadas.

Ahora, ¿cómo saber que el significado que se asocia a un código es el adecuado? El principal criterio es la coherencia del texto, es decir, la relación que tiene la significación asignada con otras significaciones. Así pues, leer es buscar una serie de significaciones que sean coherentes entre sí. Tiene que ser una búsqueda, pues no hay códigos comunes: una palabra puede significar algo en una parte y otra cosa en otra parte. Los sentidos son variados y es por ello que el lector debe indagar por los adecuados.

Que leer es trabajar, quiere decir ante todo que no hay un tal código común al que hayan sido "traducidas" las significaciones que luego vamos a descifrar. Cuando se trata, de una lectura en el sentido fuerte del término, entonces no hay un código común previo, pues el texto produce su propio código, le asigna su valor, ese es un punto importantísimo en la teoría de la lectura: no tenemos un código común, tenemos que extraer el código del texto mismo (Zuleta, 1982).

Entonces ¿Qué es lo que hace difícil leer filosofía? ¿En qué consiste su exigencia? Dicho de otro modo: ¿qué es lo que limita a los estudiantes a la hora de estudiar filosofía? Con Estanislao Zuleta, diremos que la limitación no es producida por ausencia de elementos, sino por un método inadecuado de acercarse al texto. La lectura de un texto de filosofía no es algo que se realiza en la pasividad del lector sino en su constante actividad, y como bien es sabido, trabajar (actividad) exige más que recibir (pasividad).

Y, ¿Cuál es exactamente este trabajo que se hace al leer? ¿Cuál es el método para leer? El método es pensar e interpretar. La academia, entonces, falla en su misión cuando se convierte en un espacio para la mera repetición de datos, en la mera transmisión de información. Las universidades, más que elementos, le debe dar capacidades a los

${ }^{5}$ En este punto, creímos pertinente recurrir al trabajo del filósofo colombiano Estanislao Zuleta (Zuleta, 1982). 
que entren en ellas. Capacidades que les han de formar no solo en la profesión que han escogido, sino también como personas, como ciudadanos. El deber de la academia, si desea formar ciudadanos preparados para un tiempo de diversidad, es el de enseñar a pensar, el enseñar a filosofar.

Lo anterior muestra en qué consiste aquel esfuerzo de hacer filosofía, y como se mostró arriba, este esfuerzo es el que trae consigo habilidades que forman propiamente a los ciudadanos. Entonces, si bien hay un esfuerzo, se tiene que hacer. Así pues, se ha probado la necesidad e importancia de la filosofía dentro de una sociedad y, por ende, la importancia de la filosofía en la academia. La Universidad es pues, un escenario clave para retomar a los clásicos, trabajarlos y expandir su lectura.

\section{CONCLUSIÓN}

En conclusión, es importante estudiar filosofía pues como se ha mostrado, su estudio es útil. Y es útil porque ayuda a entender y a cambiar al mundo, y porque su ejercicio mejora a la persona que la práctica. Lo que ayuda a entender del mundo es cómo es (similar a la ciencia), cómo podría ser y cómo debería ser. Entre las ventajas que trae el ejercicio filosófico cabe destacar la capacidad crítica, el mejoramiento de las formas de razonar y la creación de nuevas formas de entender la realidad. Se aprecia que la primera ventaja atañe a todas las personas especialmente en cuanto a seres políticos; la segunda ventaja nos atañe en cuanto seres racionales; y la tercera ventaja nos atañe en cuanto seres éticos y epistemológicos.

Subsecuentemente también se vio que los clásicos son importantes de leer porque nos ayudan a formarnos una imagen de la realidad, y cuando no es así, nos ayudan a cuestionar la imagen de la realidad que ya teníamos formada. Eliminar la filosofía de las aulas puede generar una sociedad acrítica, simplista, sin visión de mundo y que a duras penas le interese su entorno. Eliminar la filosofía de la academia nos dejaría una sociedad sin ciudadanos.

\section{REFERENCIAS BIBLIOGRÁFICAS}

Barletta, N. (2015). El rol del lenguaje en el aprendizaje.

Carlino, P. (2005). Escribir, leer y aprender en la universidad Una introducción a la alfabetización académica. Buenos Aires: Fondo de Cultura Económica.

Cassany, D., \& Morales, O. A. (2009). Leer y escribir en la universidad: los géneros científicos. Barcelona: Paidós. 
Ferro, J. (31 de 05 de 2015). "El hombre es una pasión inútil": Jesús Ferro Bayona. (A. Benedatti, Entrevistador) Obtenido de El Heraldo: https://www.elheraldo.co/ tendencias/el-hombre-es-una-pasion-inutil-jesus-ferro-bayona-197421

Heidegger, M. (2005). ¿Qué significa pensar? Madrid: Editorial Trotta, S.A.

Moyano, E. (2007). Enseñanza de habilidades discursivas en español en contexto preuniversitario: Una aproximación desde la LSF. Revista signos, 573-608.

Narvaja, E., Stefano, M. D., \& Pereira, C. (2004). La Lectura y la Escritura en La Universidad. Buenos Aires: UNIVERSIDAD DE BUENOS AIRES.

Nussbaum, M. C. (2005). El cultivo de la humanidad. Una defensa clásica de la reforma en la educación liberal. Barcelona: Ediciones Paidós Ibérica, S.A.

UNESCO. (2011). La Filosofía. Una Escuela de la Libertad. París: La Organización de las Naciones Unidas para la Educación, la Ciencia y la Cultura Sector de las Ciencias Sociales y Humanas.

Zuleta, E. (1982). Sobre la lectura. 